\title{
Narrative review of immunotherapy and radiation therapy in elderly patients
}

\author{
Brittney Chau ${ }^{1}$, Jonnby LaGuardia ${ }^{2}$, Caressa Hui ${ }^{3}$, Linda Ye $^{1}$, Yan Xing ${ }^{4}$, Erminia Massarelli ${ }^{4}$, \\ Arya Amini ${ }^{5}$
}

${ }^{1}$ New York Medical College, School of Medicine, NY, USA; ${ }^{2}$ University of Rochester, School of Medicine, NY, USA; ${ }^{3}$ Department of Radiation Oncology, Stanford University, CA, USA; ${ }^{4}$ Department of Medical Oncology, City of Hope National Medical Center, CA, USA; ${ }^{5}$ Department of Radiation Oncology, City of Hope National Medical Center, CA, USA

Contributions: (I) Conception and design: B Chau, J LaGuardia, C Hui, L Ye, A Amini; (II) Administrative support: A Amini; (III) Provision of study materials or patients: B Chau, J LaGuardia, C Hui, L Ye, A Amini; (IV) Collection and assembly of data: B Chau, J LaGuardia, C Hui, L Ye, A Amini; (V) Data analysis and interpretation: All authors; (VI) Manuscript writing: All authors; (VII) Final approval of manuscript: All authors.

Correspondence to: Arya Amini, MD. Department of Radiation Oncology, City of Hope National Medical Center, 1500 E Duarte Avenue, Duarte, CA, 91010, USA. Email: aamini@coh.org.

\begin{abstract}
Cancer is primarily a disease of the elderly, but there is a disproportionate lack of data from clinical trials in this population. Oncologists tend to underdiagnose and undertreat geriatric patients with cancer, leading to poor survival outcomes. New therapies or therapeutic combinations such as immunotherapy and stereotactic body radiation therapy (SBRT) can be used in the elderly for better tumor control and survival, with fewer toxicities. The aim of this review is to describe the synergistic effects of immunotherapy and radiation therapy (RT) and to discuss the use of these therapies concurrently and/ or sequentially in the elderly. To gain a fuller picture of their elderly patient's health, physicians may also consider incorporating a comprehensive geriatric assessment (CGA) to evaluate their functional capacity, cognition, physical and mental health, and social supports which we will discuss in this review. It is recommended that oncologists use geriatric assessments in their everyday practice to have better insight on their complex elderly patients, therefore providing them a higher quality of care. They should also be incorporated in clinical trials as a way to assess and include more elderly patients in the study. Ultimately, the elderly deserve to be treated with more than their chronological age in mind, and new combination therapies and use of a geriatric assessment can help achieve that.
\end{abstract}

Keywords: Radiation therapy (RT); stereotactic body radiation therapy (SBRT); immunotherapy; immune checkpoint inhibitors (ICIs); immunotherapy; elderly; older adults

Submitted Jul 31, 2020. Accepted for publication Aug 20, 2020.

doi: $10.21037 /$ tcr-20-2637

View this article at: http://dx.doi.org/10.21037/tcr-20-2637

\section{Introduction}

Cancer disproportionally affects individuals in the age 70 years and older, with the elderly population accounting for $80 \%$ of all cancer related deaths in the United States (1). As the elderly population in the United States increases, the incidence of cancer in the elderly is also expected to increase from 1.0 million in 2010 to 1.6 million in 2030, as aging is a prominent risk factor for malignancies (2). There is also an increased risk of mortality due to cancer in the elderly (3). Another challenge of treating cancer in the elderly is the underdiagnosis and undertreatment of their malignancies $(4,5)$. Elderly patients are likely to receive less than the standard of care provided to the younger population, therefore leading to worse outcomes. Older patients were more likely to receive deintensified treatment and less likely to receive curative treatment, leading to worse comorbidity and decreased 
performance status (4-6).

More innovative and focused curative treatment in the elderly are needed to help solve the problem of undertreatment and worse survival outcomes in this population. Immunotherapy and use of immune checkpoint inhibitors (ICIs) show promise in the elderly population, due to the lower rates of severe toxicities when compared to traditional cytotoxic chemotherapy (7). However, as people age, a phenomenon known as immunosenescence, leads to higher susceptibility to cancer, infections, and other autoimmune diseases. Immunosenescence causes a shift in the immune system to a pro-inflammatory phenotype via production of pro-inflammatory cytokines, which can lead to hyper-inflammatory states (8). The direct impact of immunotherapy in the elderly is largely unknown, but data from multiple trials have shown that ICIs are still highly efficacious in this population, even if there is a higher risk of toxicity when compared to younger patients $(9,10)$.

In addition to increased use of immunotherapy in the elderly, radiation therapy (RT) should be considered for this population. RT has been shown to be effective and safe, while providing good local control and low toxicity rates. It can be used as a primary treatment for local control or as palliative treatment to preserve quality of life (QoL) and improve survival. New RT techniques such as brachytherapy and stereotactic body radiotherapy (SBRT) allow for more specialized treatment of cancer in the elderly, as they apply high doses of radiation locally with maximum preservation of nearby normal structures, all within a short total treatment time (11-13). However, clinicians still need to be cautious due to the increased sensitivity of the elderly to radiation or combined chemoradiation toxicities and other treatment related burdens (14). We present the following article in accordance with the Narrative Review reporting checklist (available at http://dx.doi.org/10.21037/tcr-20-2637).

\section{Methods}

An analysis of the medical literature from peer-reviewed journals was conducted from 1970 to 2020 of the PubMed database to retrieve a comprehensive set of relevant articles. The search strategy was developed based on National Library of Medicine ${ }^{\circledR}$ Medical Subject Headings $\left(\mathrm{MeSH}^{\circledR}\right)$ with addition of subject-specific keywords. The literature was reviewed for quality of study design, cohort size, selection bias, evaluation of participants in relation to time from exposure, and methods of assessments.

\section{Combining immunotherapy and RT}

\section{Immune stimulating effects}

The synergistic effects of combining immunotherapy and RT were first described in the 1970s (15). RT has been shown to prime the antitumor response, and this reaction combined with immunotherapies can unleash a more powerful antitumor immune response (16). Immunostimulatory effects of RT include increased recruitment and action of CD8+ T cells and dendritic cells (DC) and increased activation of pro-death signaling in tumor cells through increased expression of major histocompatibility complex (MHC) class I and FAS (17). Lugade et al. showed that RT of melanoma tumors in mice models increased the number of $\mathrm{T}$ cells that secreted interferon- $\gamma(\mathrm{IFN}-\gamma)$ and proceeded to exhibit lytic activity. They showed that a single dose radiation treatment resulted in a 3 -fold increase in the activated $\mathrm{T}$ cell hybridoma B3Z. They proposed that the RT upregulated the molecule vascular cell adhesion protein 1 (VCAM-1) to create a tumor microenvironment that allowed for increased infiltration and killing of tumors by tumor antigen reactive CD8+ T cells (18). Gupta et al. found that the efficacy of a 10 Gy dose of RT depends largely on the subsequent activation of DCs and CD8+ T cells. They found that this activation of DCs was crucial to tumor control, rather than the amount of antigen presented by tumor cells (19). Other studies have demonstrated the stimulatory effects of RT on CD8+ T cells and DCs (20-24).

In addition to the increased activation of CD8+ $\mathrm{T}$ cells and DCs, the combination of RT and immunotherapy also increases presentation of MHC class I and the FAS receptor, leading to increased killing of tumor cells. The cell models of Reits et al. showed a dose-dependent increase in MHC class I expression when exposed to RT, through increased proteasome and mTOR kinase activity, resulting in an increase in intracellular peptides available for surveillance by CD8+ T cells (25). RT also stimulates the immune system through increased presentation of FAS and other tumor antigens, activating the apoptotic pathway. Chakraborty et al. found that RT enhanced FAS expression in colon carcinoma cell models and mice models. The FAS/FAS-L pathway is the primary pathway for cytotoxic $\mathrm{T}$ cell killing of cells. The upregulated FAS was biologically active and upregulation was maintained for over 96 hours. They found that localized RT significantly increased tumor rejection by cytotoxic $\mathrm{T}$ cells. In addition, in vivo mice studies showed the enhanced efficacy of cytotoxic $\mathrm{T}$ cells when exposed 
to RT. The $50 \%$ of mice treated with RT and cytotoxic T lymphocyte adoptive transfer resolved their tumor mass and were tumor free for 40 days (26). Lastly, several studies have also demonstrated these effects (27-32).

\section{Immune inbibitory effects}

In contrast to its stimulatory effects on the immune system, RT also has inhibitory effects such as increasing the expression of immune checkpoint molecules and increasing expression of regulatory T cells. Immune checkpoint molecules such as programed cell death ligand 1 (PD-L1) and cytotoxic T-lymphocyte antigen 4 (CTLA-4) are normally expressed by normal, healthy cells to stop unnecessary apoptosis from cytotoxic $\mathrm{T}$ cells but can be exploited by tumor cells to evade the immune system (33). Park et al. found that knocking out PD$\mathrm{L} 1$ in mice resulted in increased antitumor response and survival when compared to PD-L1 wild-type mice after SBRT. Combined, SBRT + PD-L1 blockade, was more effective than SBRT alone, leading to almost complete regression of the primary tumor, and $66 \%$ reduction of a secondary tumor, which was defined as disease outside of the radiation field (34). Similar synergistic effects have been shown when combining RT and anti-CTLA-4 antibodies. CTLA-4 acts similarly to PD-L1 by suppressing immune responses via negative signaling pathways and inhibition of activating signaling. Ruocco et al. found in mouse models that the combination of anti-CTLA-4 antibodies and RT increased MHC class I cytotoxic T lymphocyte killing. Combined, RT and anti-CTLA-4 antibodies successfully controlled the primary tumor and also reduced the nonirradiated metastases of the mice (35). Many studies have demonstrated similar synergism of RT with anti-PD-L1 or anti-CTLA-4 antibodies (27,34-43).

In addition to its effects on ICIs such as PDL1 and CTLA-4, RT also increases immune system inhibition by increasing the regulatory $\mathrm{T}$ cell population. Regulatory $\mathrm{T}$ cells modulate effector $\mathrm{T}$ cells by producing immunosuppressive cytokines like IL-10 and TGF- $\beta$. Tumor cells can recruit peripheral regulatory $\mathrm{T}$ cells and induce the conversion of $\mathrm{CD} 4+\mathrm{T}$ cells into regulatory $\mathrm{T}$ cells within the tumor, therefore inhibiting the proliferation and apoptotic activity of cytotoxic T cells. Beauford et al. found that human natural regulatory $\mathrm{T}$ cells and induced regulatory $\mathrm{T}$ cells are more resistant to RT than CD4+ T cells. However, the surviving regulatory $\mathrm{T}$ cells had reduced capability to decrease the proliferation of $\mathrm{CD} 8+\mathrm{T}$ cells.
This may be due to the decreased expression of FoxP3 after $\mathrm{RT}$, due to the altered gene methylation that irradiation causes. Furthermore, RT also modulates the expression of other proteins associated with regulatory $\mathrm{T}$ cells, such as CD25, CD73, and LAG-3. CD25 expression is regulated by FoxP3 and was decreased with RT. CD73 expression increased with RT, and Beauford et al. hypothesized this was due to an increase in TGF- $\beta$, although another unknown mechanism may also be involved, since CD39, another protein sensitive to TGF- $\beta$, was not increased. Finally, LAG-3 expression was significantly increased after exposure to RT. LAG-3 expression on regulatory T cells has been shown to confer greater suppressive capacity, but the results of these experiments showed the opposite, indicating that other signals from proteins such as MHC class II from other immune cells are needed to maximize the suppressive capability of LAG-3 + regulatory T cells (44). Although research on the effects of $\mathrm{RT}$ on regulatory $\mathrm{T}$ cells is controversial and can show different results, the resistance of regulatory $\mathrm{T}$ cells to $\mathrm{RT}$ has been demonstrated in several other studies (45-48).

\section{RT and immunotherapy in the elderly}

\section{Immunotherapy in the elderly}

While the use of immunotherapy has increased in recent times because of its high efficacy and low toxicity compared to cytotoxic chemotherapy, the use of immunotherapy in the elderly has not been studied well compared to their younger counterparts. Elderly adults are under-represented in clinical trials, and because their immune system undergoes immunosenescence, it is hard to extrapolate results of studies and clinical trials of younger patients to an older population. Hallmarks of immunosenescence include a lower number of naive CD8+ and CD4+ T cells in the peripheral blood, likely due to depletion of naive $\mathrm{T}$ cells by a lifetime's exposure to immune pathogens (49). In addition, the number of DCs decline, while the amount of mature, differentiated CD8+ T cells increases with age. This leads to a decrease in $\mathrm{T}$ cell function, which can cause an impaired response to ICIs (50). Immunosenescence is thought to be associated with the increased prevalence of cancer and other age-related diseases in the elderly population. Another phenomenon known as "inflamm-aging" occurs due to increased amounts of inflammatory cytokines caused by immunosenescence $(9,51)$.

Many ICIs targeting CTLA-4, PD-1, or PD-L1 have 
been approved for the treatment of metastatic melanoma, advanced non-small cell lung cancer (NSCLC), renal cell cancer (RCC), and head and neck cancer with agents like pembrolizumab and nivolumab (52,53). ICIs enhance the body's immune response against tumor cells but can also lead to immune-related adverse events (IRAEs), which are caused by activated $\mathrm{T}$ cells infiltrating normal tissues, made more severe by blocking immune checkpoints (54). While serious IRAEs are rare $(<1 \%)$, they may be more challenging to treat in the elderly due to age-associated comorbidities and reduced functional reserve (51). In several clinical trials, there have been no significant difference in the incidence of IRAEs between patients $>65$ and $<65$ years. An overview of toxicities of ICIs in elderly patients by Alkharabsheh et al. generally saw no overall difference in safety between young and elderly patients in the KEYNOTE and Checkmate trials, and other trials testing atezolizumab or durvalumab (55-61). However, retrospective data from Memorial Sloan Kettering Cancer Center showed higher rates of discontinuation and increased IRAEs in melanoma patients over 80 years of age being treated with ICIs (55). A metaanalysis of nine randomized controlled trials by Nishijima et al. showed improved overall survival (OS) and progression free survival (PFS) in younger and older patients treated with ICIs. However, for patients $\geq 75$ years of age, there was no OS benefit found when treated with anti-PD-L1 monoclonal antibody (mAb). The authors concluded that this could be due to immunosenescence or lack of enough statistical power to show a significant difference (62).

A retrospective, pooled analysis of the KEYNOTE studies by Nosaki et al. found that pembrolizumab, a PD$\mathrm{L} 1 \mathrm{mAb}$, was associated with improved OS in patients with NSCLC $\geq 75$ years of age when compared to platinumbased chemotherapy [hazard ratio (HR), 0.41, 95\% CI: $0.23-0.73$ ] when used as a first line therapy. The KEYNOTE clinical trials studied 2,348 patients with PD-L1+ NSCLC, 264 of who were elderly, defined as $\geq 75$ years of age. Treatment with pembrolizumab improved OS for both elderly and younger patients when compared to chemotherapy. Pembrolizumab was also associated with fewer overall treatment-related adverse effects in this population (68.5\% vs. $94.3 \%)$ compared to chemotherapy. The incidence of IRAEs or infusion reactions was higher in elderly patients treated with pembrolizumab compared to chemotherapy ( $24.8 \%$ vs. $6.77 \%$ ), but this incidence of IRAEs in pembrolizumab $v s$. chemotherapy was comparable to that of the younger population ( $25 \%$ vs. $5.9 \%)$ (63-66). Marur et al. performed a similar analysis of four clinical trials testing ICI use for patients with advanced or metastatic NSCLC with disease progression after platinumbased chemotherapy. A total of 259 out of 2,824 patients enrolled in these trials were elderly ( $\geq 75$ years). Patients receiving anti-PD-1/PD-L1 mAbs had increased OS compared to chemotherapy in all age groups. In addition, grade 3 or 4 treatment-related adverse effects with anti$\mathrm{PD}-1 / \mathrm{PD}-\mathrm{L} 1 \mathrm{mAbs}$ were less frequent in patients $\geq 75$ years (23\%) when compared to patients $65-74$ years (49\%) and $<65$ years (47\%) (67). Overall, this data and other studies support the use of pembrolizumab as monotherapy in elderly patients with PD-L1 >0\% NSCLC, as it has a better OS and more favorable safety profile when compared to chemotherapy (63,67-71).

Melanoma, another highly immunogenic cancer like NSCLC, has also been revolutionized by treatment with ICIs. A retrospective study by Betof $e t$ al. studied 254 patients from two academic centers with metastatic melanoma, treated with anti-PD-L1 or anti-PD-1 mAbs. There were 65 patients aged $65-74$ and 47 patients $\geq 75$ years of age. They found that there was no significant difference in the OS, PFS, and some toxicities such as colitis, hepatitis, and pneumonitis between the older and younger patient populations. The incidence of other toxicities, including arthritis and thyroiditis were higher in patients aged $65-74$ years and $\geq 75$ years, respectively. Arthritis was the only significantly increased immunotoxicity in the elderly (10.8\%, P=0.02) (72). Cybulska-Stopa et al. studied 318 patients with non-resectable or metastatic melanoma treated with anti-CTLA-4 and/or anti-PD-1 mAbs. Eighty-two patients $\geq 70$ years were included in the study, and comorbidities including hypertension, cardiovascular disease, and diabetes were present in $84 \%$ of patients $\geq 70$ years of age. Cybulska-Stopa et al. found very similar results to Betof et al., with no significant difference in 2-year OS or PFS, and toxicities between patients aged $<70$ and $>70$ years. Additionally, the presence of comorbidities in patients $\geq 70$ years of age was not significantly associated with an increased risk of toxicity $(\mathrm{P}=0.790)(73)$. Overall, the results of these studies and more show that the use of ICIs should not be limited in the elderly, even those with comorbidities, but close monitoring should be used in these patients regardless (72-77).

\section{RT in the elderly}

$\mathrm{RT}$ is a primary component in the treatment of elderly cancer patients with solid tumors and is a potentially 
curative option for older patients unable to undergo surgery. Advances to the field allowing for lower toxicity and increased efficacy have been made, allowing elderly patients to more easily undergo RT. However, elderly patients are underrepresented in randomized clinical trials for RT, similar to their previously discussed underrepresentation with immunotherapies. More studies focused specifically on this population would be beneficial for extrapolating data from trials into the actual patient population (78). Additionally, oncologists may exhibit unconscious bias against the elderly and can be more reluctant to treat an older cancer patient aggressively because of their age (79). However, with modern advancements such as SBRT and brachytherapy, curative treatment for elderly patients may be feasible, even if they have multiple comorbidities or frailty that excludes surgery or chemotherapy (13). Therefore, the use of RT should not be discounted in the elderly and has the potential to be more than a palliative option.

As with all other cancer therapies, RT does not come without toxicities. RT can cause acute or late radiation toxicity that can affect treatment plans and outcomes. Acute toxicity is due to damage to rapidly self-proliferating tissues such as skin or mucosa and normally appears 2 -3 weeks after starting treatment. The damage can force treatment to stop to allow the tissue to recover, but this pause can allow for the accelerated proliferation of cancer cells, decreasing the likelihood of good local control. On the other hand, late toxicity is caused by damage to tissues that turn over more slowly, such as vascular and connective tissue. Late toxicities can appear months to years after RT and can decrease QoL of the patient because of their irreversible and progressive nature. In addition, elderly patients may have decreased functional reserve of their organs, leading to increased toxicity even with low doses of radiation $(80,81)$. Retrospective studies have shown that the tolerance to toxicities such as mucositis is decreased in the elderly when compared to younger patients. The elderly are more vulnerable to toxicities and the complications they can cause, such as dehydration $(82,83)$. In addition, the concomitant addition of chemotherapy to RT leads to increased toxicity in patients $\geq 70$ years, leading to increased hospitalizations and worse OS (84). Caution should be used when treating elderly patients, but this does not always call for a less than curative treatment plan.

In patients with NSCLC, SBRT has been shown to be comparable to surgery and is the treatment of choice for elderly patients with multiple comorbidities that exclude surgery. A review by Nguyen et al. concluded that SBRT was well tolerated by elderly patients with NSCLC and resulted in similar survival rates as those of surgery treatment. Comorbidities such as hypertension, diabetes, and renal disease may increase complication rates after surgery, leading to decreased patient survival after surgical resection. The 3-year OS in elderly patients who underwent SBRT ranged from $40.7 \%$ to $53 \%$, even in patients with high Charlson comorbidity grades. SBRT in elderly patients achieved excellent local control, ranging from $83 \%$ to $100 \%$ with minimal morbidity, and low occurrence of serious toxicities $(2.1 \%$ to $10 \%)$. In comparison, with surgical resection, $44 \%$ of patients had a grade 3 complication and one patient died from surgical complications (85). A retrospective study by Takeda et al. included 109 patients $\geq 80$ years with NSCLC who were all treated with SBRT. The 1 -year OS was $95.0 \%$ and the 3 -year OS was $53.7 \%$ for all patients. After one month of SBRT, 90 patients experienced grade $0-1$ radiation pneumonitis, 13 patients grade 2, 4 patients grade 3, 0 patients grade 4, and 1 patient grade 5. After SBRT, QoL was maintained, and emotional functioning was improved, whereas QoL worsened after surgery (86). Several studies report similar findings regarding the better or comparable outcomes of SBRT against surgery in elderly patients with NSCLC (87-92).

Head and neck squamous cell carcinoma (HNSCC) is another cancer frequently treated with RT. Haehl et al. analyzed 246 patients $\geq 65$ years of age with HNSCC who received chemoradiotherapy or RT alone. Out of these patients, 166 underwent definitive RT and the other 80 adjuvant chemoradiotherapy. Patients aged 65-74 years had significantly better OS when treated with concomitant chemoradiotherapy, although the data may be skewed due to the low number of patients $\geq 75$ years undergoing chemoradiotherapy. Additionally, this study reported $56.1 \%$ of patients reporting grade $3 / 4$ toxicities (93). Another study by Sommers et al. described 674 patients with HNSCC treated with definitive RT, in which elderly patients had worse OS than their younger counterparts. The worse survival outcomes were mainly a result of increased non-cancer-related mortality and comorbidities (94). However, Bonomo et al. found that a hypofractionated radiation schedule provides good clinical benefit with low toxicity in elderly patients with locally advanced HNSCC. These patients were unable to undergo potentially curative adjuvant chemoradiotherapy or highdose RT due to their frailty and comorbidities, therefore a hypofractionated RT schedule worked best for them (95). 
For patients able to tolerate combined chemoradiotherapy, Belgioia et al. recommends they be treated with this method, due to better loco-regional control rates when compared to patients treated with RT only. When RT is used alone, an average weekly radiation dose $>9.236 \mathrm{~Gy}$ is recommended for the elderly in order to improve local control of the tumor (96). Additionally, a study by Amini et al. found increased OS for HNSCC patients $\leq 81$ years with low comorbidity scores and $\mathrm{T} 1-2 / \mathrm{N} 2-3$ or $\mathrm{T} 3-4 / \mathrm{N} 0-3$ disease, when treated with concurrent chemoradiation, compared to RT alone. Patients who were older than 81, who had T1-2/N1 and Charlson-Deyo comorbidity score 0-1 (CD0-1) disease, or who had T3-4/N1+ and CD1+ disease did not have increased survival with chemoradiation. Elderly patients able to tolerate chemoradiation or who have disease receptive to it should be treated this way for increased OS and better tumor local control (97). Overall, RT is a good choice for elderly patients, especially those with increased frailty or many comorbidities. When treating the elderly with RT, a balance between caution and aggression is needed for them to maintain their QoL while positively affecting survival outcomes.

\section{Combining immunotherapy and RT in the elderly}

The combination of immunotherapy and RT has been well studied in clinical trials and is a good option for younger populations but is not well studied in the elderly. The combination allows for increased immune activation because ICIs can combat the immune suppressive qualities of RT, as discussed previously. When treating older patients with combination therapies, there is concern for increased risk of toxicity compared to treatment with single therapies, due to their decreased functional capacity and increased comorbidities (98). However, the combination could be used to maximize the efficacy of each arm of treatment in order to achieve a curative or therapeutic outcome, rather than a palliative one (16). In particular, the combination of SBRT and targeted immunotherapy has been shown to improve disease control rates in oligometastatic disease (99). Therefore, if patients are able to tolerate the combination of immunotherapy + RT, this treatment can be crucial to overcoming tumor immunoresistance and aid in patient survival (100).

A comprehensive review by Belgioia et al. describes several studies in which elderly patients were treated with RT and targeted therapy or immunotherapy concomitantly or sequentially. The review focused more on targeted therapies, but did look at studies where ICIs such as ipilimumab (anti-CTLA-4) and pembrolizumab (anti-PD-1) were combined with RT. The ipilimumab + RT combination was mainly used for elderly patients with metastatic or advanced melanoma, NSCLC, or castration-resistant prostate cancer. In these groups, RT and ipilimumab combined improved outcomes with no increased toxicities. Melanoma patients with brain metastases had comparable survival with patients without brain metastases when treated with ipilimumab and SBRT. The combination was also tolerated well in NSCLC and prostate cancer patients (98).

Belgioia et al. also looked at pembrolizumab, an antiPD-1 therapy, combined with RT in elderly patients with NSCLC and unresectable oral cavity squamous cell carcinoma (OCSCC). In NSCLC, pembrolizumab + RT lead to a significantly longer OS and PFS with a slightly higher risk of pneumonitis according to case reports. A case report of an elderly women with OCSCC suggests that the synergistic effect of pembrolizumab and RT lead to the excellent clinical response of her unresectable recurrent cancer (98). In addition, a case report by Lazzari et al. describes an elderly patient with refractory advanced NSCLC who achieved complete remission after being treated with nivolumab (anti-PD-1) after a course of RT. The patient was first treated with cisplatin and vinorelbine chemotherapy, which resulted in the disappearance of two pulmonary nodules, with a third nodule having increased in size. The growing third nodule received consolidative RT with nivolumab monotherapy, which resulted in complete resolution of the pulmonary lesion and was well tolerated, with pruritus as the main side effect (100). The study is an example of combination RT and immunotherapy in patients with oligoprogressive disease and ongoing studies are currently evaluating this clinical scenario.

Another type of immunotherapy + RT combination therapy that has been studied in the elderly is adoptive cytokine-induced killer cell (CIK) or DC therapy with RT. In a study by Yan et al., 68 elderly patients with esophageal carcinoma were randomized into a control group that received RT only or DC-CIK immunotherapy + RT. They found a significant increase in treatment efficacy of the combination immunotherapy + RT. The patients in the study group had better complete and partial control, as well as better stability of the cancer and less tumor progression. The objective response rate in the study group and the control group was $41.2 \%$ and $29.4 \%$ respectively, and the disease control rate was $85.3 \%$ and $61.7 \%$ respectively. The control group had higher rates of locoregional and distant 
progression, though this was not statistically significant. Toxicities varied between the two groups, with the study group reporting significantly more fever, overexcitation, and insomnia, and the control group reporting significantly more severe bone marrow suppression. Digestive tract reactions and tracheitis were observed in both groups. The study concludes that DC-CIK immunotherapy and IMRT is safe and effective in treating elderly patients with esophageal carcinoma (101). Overall, these studies support the combination of immunotherapy and RT in the elderly, but more higher powered studies are need for our older adults.

\section{Using geriatric assessments}

The increased incidence of cancer in the elderly is disproportionate to the paucity of data and clinical trials focused on treating cancer in this multifaceted population. Clinicians may have trouble treating the elderly because of this lack of data or because of inadequate training and understanding of the elderly, leading to undertreatment and potentially worse outcomes (14). A comprehensive geriatric assessment (CGA) can help physicians better understand the complexity of older patients. A CGA is a multidisciplinary evaluation of an elderly patient's functional status, medical comorbidities, cognition, psychological state, nutritional status, and social support. The CGA is able to predict survival, toxicities, morbidity, and mortality of an elderly cancer patient, and incorporating it into the general practice of treating elderly cancer patients can guide treatment plans to improve survival and QoL outcomes (102). The elderly population's representation in clinical trials is biased due to selection of relatively healthy elderly individuals to meet clinical trial inclusion criteria. Clinical trials generally require their selected patients to have little to no comorbidities, which is not representative of the general elderly population $(69,78)$. Clinical trials should consider using assessments such as the CGA to more properly measure whether or not an elderly person is able to be included in the study, as our current performance scores are not felt to properly capture the true functional status of our older adult patients. Furthermore, a CGA can provide additional endpoints for clinical trials such as QoL and functional independence to expand the breadth of information the trial provides (102).

It is important for current physicians to be able to use tools such as the CGA. A study by Morris et al. of radiation oncologist trainees identified knowledge gaps regarding geriatric oncology, with $91.8 \%$ of respondents never receiving specific geriatric oncology training, and $80.3 \%$ of trainees rarely or never using geriatric assessment tools. Due to this lack of knowledge, trainees had little confidence in their ability to manage complex issues in the elderly. The $85.3 \%$ of trainees agreed or strongly agreed that they needed more geriatric oncology specific training (103). Future physicians should be trained to use assessments such as the CGA to provide personalized and adequate care for their elderly patients. A CGA is able to identify vulnerabilities or abnormalities in the elderly that are not addressed in routine oncology visits and performance status scores including the European Cooperative Oncology Group (ECOG) and Karnofsky Performance Status (KPS) scores. This helps find areas where interventions can be made outside of oncologic treatment, such as nutrition, social support, and physical therapy (104). In addition, geriatric assessment tools can be used to better identify frailty, an increased vulnerability to negative changes in health status. A study by Kirkhus et al. found that a geriatric assessment was better able to identify frailty in older cancer patients than oncologists' clinical judgement of frailty. Therefore, using a geriatric assessment helps oncologists make appropriate treatment plans for their elderly patients (105). In order to provide elderly cancer patients with the highest quality of care, it is recommended that all oncologists use some form of geriatric assessment in their clinical practice and prospective studies including older adults should make CGA a component of the studies.

\section{Conclusions}

The elderly continue to be an understudied population despite their increased incidence of cancer and other comorbidities. The revolutionizing combination of RT and immunotherapy may prove to be particularly helpful in this population, due to their combined immune stimulatory effects and relatively tolerable side effect profile. There is some concrete data on the use of RT or immunotherapy separately in the elderly, but a substantial lack of information on the combination of the two in the eerly. A solution to the underrepresentation of this population in clinical trials and other studies is increased use of geriatric assessments by clinicians and researchers. Geriatric assessments help synthesize information such as functional status, comorbidities, and social support in order to help physicians look at elderly patients as more than their chronological age. They help physicians be more aware 
of their elderly patients' functional status and give them a clear path to high-quality and appropriate treatments for their patients. Ultimately, the elderly are a complex and heterogenous population, and should be treated as all cancer patients, with a multidisciplinary approach.

\section{Acknowledgments}

Funding: None.

\section{Footnote}

Provenance and Peer Review: This article was commissioned by the editorial office, Translational Cancer Research for the series "Synergy in Action: Novel Approaches to Combining Radiation Therapy and Immunotherapy". The article has undergone external peer review.

Reporting Checklist: The authors have completed the Narrative Review reporting checklist. Available at http:// dx.doi.org/10.21037/tcr-20-2637

Conflicts of Interest: All authors have completed the ICMJE uniform disclosure form (available at http://dx.doi. org/10.21037/tcr-20-2637). The series "Synergy in Action: Novel Approaches to Combining Radiation Therapy and Immunotherapy" was commissioned by the editorial office without any funding or sponsorship. AA served as the unpaid Guest Editor of the series and serves as an unpaid editorial board member of Translational Cancer Research from Dec 2019 to Nov 2021. The authors have no other conflicts of interest to declare.

Ethical Statement: The authors are accountable for all aspects of the work in ensuring that questions related to the accuracy or integrity of any part of the work are appropriately investigated and resolved.

Open Access Statement: This is an Open Access article distributed in accordance with the Creative Commons Attribution-NonCommercial-NoDerivs 4.0 International License (CC BY-NC-ND 4.0), which permits the noncommercial replication and distribution of the article with the strict proviso that no changes or edits are made and the original work is properly cited (including links to both the formal publication through the relevant DOI and the license). See: https://creativecommons.org/licenses/by-nc-nd/4.0/.

\section{References}

1. Zeber JE, Copeland LA, Hosek BJ, et al. Cancer rates, medical comorbidities, and treatment modalities in the oldest patients. Crit Rev Oncol Hematol 2008;67:237-42.

2. Smith BD, Smith G, Hurria A, et al. Future of cancer incidence in the United States: burdens upon an aging, changing nation. J Clin Oncol 2009;27:2758-65.

3. Misra D, Seo Ph, Cohen HJ. Aging and cancer. Clin Adv Hematol Oncol 2004;2:457-65.

4. Rao VS, Jameel JK, Mahapatra TK, et al. Surgery is associated with lower morbidity and longer survival in elderly breast cancer patients over 80 . Breast J 2007;13:368-73.

5. Bellury LM, Ellington L, Beck SL, et al. Elderly cancer survivorship: an integrative review and conceptual framework. Eur J Oncol Nurs 2011;15:233-42.

6. Fang P, He W, Gomez DR, et al. Influence of Age on Guideline-Concordant Cancer Care for Elderly Patients in the United States. Int J Radiat Oncol Biol Phys 2017;98:748-57.

7. Godby RC, Johnson DB, Williams GR. Immunotherapy in Older Adults with Cancer. Curr Oncol Rep 2019;21:56.

8. Prcina M, Novak M, Cigankova V, et al. Immunosenescence - the role in the immunotherapy of older population. Bratisl Lek Listy 2018;119:217-20.

9. Elias R, Hartshorn K, Rahma O, et al. Aging, immune senescence, and immunotherapy: A comprehensive review. Semin Oncol 2018;45:187-200.

10. Elias R, Morales J, Rehman Y, et al. Immune Checkpoint Inhibitors in Older Adults. Curr Oncol Rep 2016;18:47.

11. Lancellotta V, Kovács G, Tagliaferri L, et al. Age Is Not a Limiting Factor in Interventional Radiotherapy (Brachytherapy) for Patients with Localized Cancer. Biomed Res Int 2018;2018:2178469.

12. Colloca G, Tagliaferri L, Capua BD, et al. Management of The Elderly Cancer Patients Complexity: The Radiation Oncology Potential. Aging Dis 2020;11:649-57.

13. Popescu T, Karlsson U, Vinh-Hung V, et al. Challenges Facing Radiation Oncologists in The Management of Older Cancer Patients: Consensus of The International Geriatric Radiotherapy Group. Cancers (Basel) 2019;11:371.

14. Chang S, Goldstein NE, Dharmarajan KV. Managing an Older Adult with Cancer: Considerations for Radiation Oncologists. Biomed Res Int 2017;2017:1695101.

15. Milas L, Hunter N, Withers HR. Combination of 
local irradiation with systemic application of anaerobic corynebacteria in therapy of a murine fibrosarcoma. Cancer Res 1975;35:1274-7.

16. Crittenden M, Kohrt H, Levy R, et al. Current clinical trials testing combinations of immunotherapy and radiation. Semin Radiat Oncol 2015;25:54-64.

17. Pitroda SP, Chmura SJ, Weichselbaum RR. Integration of radiotherapy and immunotherapy for treatment of oligometastases. Lancet Oncol 2019;20:e434-42.

18. Lugade AA, Moran JP, Gerber SA, et al. Local radiation therapy of B16 melanoma tumors increases the generation of tumor antigen-specific effector cells that traffic to the tumor. J Immunol 2005;174:7516-23.

19. Gupta A, Probst HC, Vuong V, et al. Radiotherapy promotes tumor-specific effector CD8+ T cells via dendritic cell activation. J Immunol 2012;189:558-66.

20. Lee Y, Auh SL, Wang Y, et al. Therapeutic effects of ablative radiation on local tumor require $\mathrm{CD} 8+\mathrm{T}$ cells: changing strategies for cancer treatment. Blood 2009;114:589-95.

21. Draghiciu O, Walczak M, Hoogeboom BN, et al. Therapeutic immunization and local low-dose tumor irradiation, a reinforcing combination. Int J Cancer 2014;134:859-72.

22. Demaria S, Bhardwaj N, McBride WH, et al. Combining radiotherapy and immunotherapy: a revived partnership. Int J Radiat Oncol Biol Phys 2005;63:655-66.

23. Vendetti FP, Karukonda P, Clump DA, et al. ATR kinase inhibitor AZD6738 potentiates CD8+ T cell-dependent antitumor activity following radiation. J Clin Invest 2018;128:3926-40.

24. Wieland A, Kamphorst AO, Adsay NV, et al. T cell receptor sequencing of activated CD8 $\mathrm{T}$ cells in the blood identifies tumor-infiltrating clones that expand after PD-1 therapy and radiation in a melanoma patient. Cancer Immunol Immunother 2018;67:1767-76.

25. Reits EA, Hodge JW, Herberts CA, et al. Radiation modulates the peptide repertoire, enhances $\mathrm{MHC}$ class I expression, and induces successful antitumor immunotherapy. J Exp Med 2006;203:1259-71.

26. Chakraborty M, Abrams SI, Camphausen K, et al. Irradiation of tumor cells up-regulates Fas and enhances CTL lytic activity and CTL adoptive immunotherapy. J Immunol 2003;170:6338-47.

27. Durgeau A, Virk Y, Corgnac S, et al. Recent Advances in Targeting CD8 T-Cell Immunity for More Effective Cancer Immunotherapy. Front Immunol 2018;9:14.

28. Ishikawa E, Tsuboi K, Saijo K, Saijo K, et al. X-irradiation to human malignant glioma cells enhances the cytotoxicity of autologous killer lymphocytes under specific conditions. Int J Radiat Oncol Biol Phys 2004;59:1505-12.

29. Weichselbaum RR, Liang H, Deng L, et al. Radiotherapy and immunotherapy: a beneficial liaison? Nat Rev Clin Oncol 2017;14:365-79.

30. Garnett CT, Palena C, Chakraborty M, et al. Sublethal irradiation of human tumor cells modulates phenotype resulting in enhanced killing by cytotoxic $\mathrm{T}$ lymphocytes. Cancer Res 2004;64:7985-94.

31. Sharabi AB, Lim M, DeWeese TL, et al. Radiation and checkpoint blockade immunotherapy: radiosensitisation and potential mechanisms of synergy. Lancet Oncol 2015;16:e498-509.

32. Ko EC, Formenti SC. Radiation therapy to enhance tumor immunotherapy: a novel application for an established modality. Int J Radiat Biol 2019;95:936-9.

33. Manukian G, Bar-Ad V, Lu B, et al. Combining Radiation and Immune Checkpoint Blockade in the Treatment of Head and Neck Squamous Cell Carcinoma. Front Oncol 2019;9:122.

34. Park SS, Dong H, Liu X, et al. PD-1 Restrains Radiotherapy-Induced Abscopal Effect. Cancer Immunol Res 2015;3:610-9.

35. Ruocco MG, Pilones KA, Kawashima N, et al. Suppressing $\mathrm{T}$ cell motility induced by anti-CTLA-4 monotherapy improves antitumor effects. J Clin Invest 2012;122:3718-30.

36. Gong J, Le TQ, Massarelli E, et al. Radiation therapy and PD-1/PD-L1 blockade: the clinical development of an evolving anticancer combination. J Immunother Cancer 2018;6:46.

37. Zeng J, See AP, Phallen J, et al. Anti-PD-1 blockade and stereotactic radiation produce long-term survival in mice with intracranial gliomas. Int J Radiat Oncol Biol Phys 2013;86:343-9.

38. Xia L, Liu Y, Wang Y. PD-1/PD-L1 Blockade Therapy in Advanced Non-Small-Cell Lung Cancer: Current Status and Future Directions. Oncologist 2019;24:S31-41.

39. Demaria S, Kawashima N, Yang AM, et al. Immunemediated inhibition of metastases after treatment with local radiation and CTLA-4 blockade in a mouse model of breast cancer. Clin Cancer Res 2005;11:728-34.

40. Pilones KA, Kawashima N, Yang AM, et al. Invariant natural killer $\mathrm{T}$ cells regulate breast cancer response to radiation and CTLA-4 blockade. Clin Cancer Res 2009; 15:597-606.

41. Derer A, Frey B, Fietkau R, et al. Immune-modulating 
properties of ionizing radiation: rationale for the treatment of cancer by combination radiotherapy and immune checkpoint inhibitors. Cancer Immunol Immunother 2016;65:779-86.

42. Formenti SC, Rudqvist NP, Golden E, et al. Radiotherapy induces responses of lung cancer to CTLA-4 blockade. Nat Med 2018;24:1845-51.

43. Deng L, Liang H, Burnette B, et al. Irradiation and antiPD-L1 treatment synergistically promote antitumor immunity in mice. J Clin Invest 2014;124:687-95.

44. Beauford SS, Kumari A, Garnett-Benson C. Ionizing radiation modulates the phenotype and function of human CD4+ induced regulatory T cells. BMC Immunol 2020;21:18.

45. Qu Y, Jin S, Zhang A, et al. Gamma-ray resistance of regulatory CD4+CD25+Foxp3+T cells in mice. Radiat Res 2010;173:148-57.

46. Qu Y, Zhang B, Liu S, et al. 2-Gy whole-body irradiation significantly alters the balance of CD4+ CD25- T effector cells and CD4+ CD25+ Foxp3+ T regulatory cells in mice. Cell Mol Immunol 2010;7:419-27.

47. Muroyama Y, Nirschl TR, Kochel CM, et al. Stereotactic Radiotherapy Increases Functionally Suppressive Regulatory T Cells in the Tumor Microenvironment. Cancer Immunol Res 2017;5:992-1004.

48. Son CH, Bae JH, Shin DY, et al. Combination effect of regulatory T-cell depletion and ionizing radiation in mouse models of lung and colon cancer. Int J Radiat Oncol Biol Phys 2015;92:390-8.

49. Pawelec G. Immunosenescence and cancer. Biogerontology 2017;18:717-21.

50. van Holstein Y, Kapiteijn E, Bastiaannet E, et al. Efficacy and Adverse Events of Immunotherapy with Checkpoint Inhibitors in Older Patients with Cancer. Drugs Aging 2019;36:927-38.

51. Hong H, Wang Q, Li J, et al. Aging, Cancer and Immunity. J Cancer 2019;10:3021-7.

52. Ferris RL, Blumenschein G Jr, Fayette J, et al. Nivolumab for Recurrent Squamous-Cell Carcinoma of the Head and Neck. N Engl J Med 2016;375:1856-67.

53. Bauml J, Seiwert TY, Pfister DG, et al. Pembrolizumab for Platinum- and Cetuximab-Refractory Head and Neck Cancer: Results From a Single-Arm, Phase II Study. J Clin Oncol 2017;35:1542-9.

54. Helissey C, Vicier C, Champiat S. The development of immunotherapy in older adults: New treatments, new toxicities? J Geriatr Oncol 2016;7:325-33.

55. Alkharabsheh O, Kannarkatt P, Kannarkatt J, et al. An overview of the toxicities of checkpoint inhibitors in older patients with cancer. J Geriatr Oncol 2018;9:451-58.

56. Robert C, Long GV, Brady B, et al. Nivolumab in previously untreated melanoma without BRAF mutation. N Engl J Med 2015;372:320-30.

57. Robert C, Schachter J, Long GV, et al. Pembrolizumab versus ipilimumab in advanced melanoma. N Engl J Med 2015;372:2521-32.

58. Wolchok JD, Kluger H, Callahan MK, et al. Nivolumab plus ipilimumab in advanced melanoma. N Engl J Med 2013;369:122-33.

59. Postow MA, Chesney J, Pavlick AC, et al. Nivolumab and ipilimumab versus ipilimumab in untreated melanoma. $\mathrm{N}$ Engl J Med 2015;372:2006-17.

60. Hodi FS, O'Day SJ, McDermott DF, et al. Improved survival with ipilimumab in patients with metastatic melanoma. N Engl J Med 2010;363:711-23.

61. Larkin J, Chiarion-Sileni V, Gonzalez R, et al. Combined nivolumab and ipilimumab or monotherapy in untreated melanoma. N Engl J Med 2015;373:23-34.

62. Nishijima TF, Muss HB, Shachar SS, et al. Comparison of efficacy of immune checkpoint inhibitors (ICIs) between younger and older patients: A systematic review and metaanalysis. Cancer Treat Rev 2016;45:30-7.

63. Nosaki K, Saka H, Hosomi Y, et al. Safety and efficacy of pembrolizumab monotherapy in elderly patients with PDL1-positive advanced non-small-cell lung cancer: Pooled analysis from the KEYNOTE-010, KEYNOTE-024, and KEYNOTE-042 studies. Lung Cancer 2019;135:188-95.

64. Reck M, Rodríguez-Abreu D, Robinson AG, et al. Pembrolizumab versus Chemotherapy for PD-L1Positive Non-Small-Cell Lung Cancer. N Engl J Med 2016;375:1823-33.

65. Herbst RS, Baas P, Kim DW, et al. Pembrolizumab versus docetaxel for previously treated, PD-L1-positive, advanced non-small-cell lung cancer (KEYNOTE-010): a randomised controlled trial. Lancet 2016;387:1540-50.

66. Mok TSK, Wu YL, Kudaba I, et al. Pembrolizumab versus chemotherapy for previously untreated, PD-L1expressing, locally advanced or metastatic non-small-cell lung cancer (KEYNOTE-042): a randomised, open-label, controlled, phase 3 trial. Lancet 2019;393:1819-30.

67. Marur S, Singh H, Mishra-Kalyani P, et al. FDA analyses of survival in older adults with metastatic non-small cell lung cancer in controlled trials of PD-1/PD-L1 blocking antibodies. Semin Oncol 2018;45:220-5.

68. Chan J, Kanesvaran R. The role of immune checkpoint inhibitors (ICI) in the treatment of metastatic non- 
small cell lung carcinoma in the elderly. Ann Transl Med 2019;7:S383.

69. Gridelli C, Sgambato A. Elderly patients and PDL1-positive advanced non-small cell lung cancer: is pembrolizumab monotherapy effective and safe? Ann Transl Med 2019;7:S282.

70. Sgambato A, Casaluce F, Gridelli C. The role of checkpoint inhibitors immunotherapy in advanced nonsmall cell lung cancer in the elderly. Expert Opin Biol Ther 2017; 17:565-71.

71. Ferrara R, Mezquita L, Auclin E, et al. Immunosenescence and immunecheckpoint inhibitors in non-small cell lung cancer patients: Does age really matter? Cancer Treat Rev 2017;60:60-8.

72. Betof AS, Nipp RD, Giobbie-Hurder A, et al. Impact of Age on Outcomes with Immunotherapy for Patients with Melanoma. Oncologist 2017;22:963-71.

73. Cybulska-Stopa B, Ługowska I, Jagodzi ska-Mucha P, et al. Immune checkpoint inhibitors therapy in older patients $(\geq$ 70 years) with metastatic melanoma: a multicentre study. Postepy Dermatol Alergol 2019;36:566-71.

74. Bastiaannet E, Battisti N, Loh KP, et al. Immunotherapy and targeted therapies in older patients with advanced melanoma; Young International Society of Geriatric Oncology review paper. J Geriatr Oncol 2019;10:389-97.

75. Chiarion Sileni V, Pigozzo J, Ascierto PA, et al. Efficacy and safety of ipilimumab in elderly patients with pretreated advanced melanoma treated at Italian centres through the expanded access programme. J Exp Clin Cancer Res 2014;33:30.

76. Ibrahim T, Mateus C, Baz M, et al. Older melanoma patients aged 75 and above retain responsiveness to anti-PD1 therapy: results of a retrospective singleinstitution cohort study. Cancer Immunol Immunother 2018;67:1571-8.

77. Perier-Muzet M, Gatt E, Péron J, et al. Association of Immunotherapy With Overall Survival in Elderly Patients With Melanoma. JAMA Dermatol 2018;154:82-7.

78. Kunkler IH, Audisio R, Belkacemi Y, et al. Review of current best practice and priorities for research in radiation oncology for elderly patients with cancer: the International Society of Geriatric Oncology (SIOG) task force. Ann Oncol 2014;25:2134-46.

79. Pang A, Ho S, Lee SC. Cancer physicians' attitude towards treatment of the elderly cancer patient in a developed Asian country. BMC Geriatr 2013;13:35.

80. Gomez-Millan J. Radiation therapy in the elderly: more side effects and complications? Crit Rev Oncol Hematol
2009;71:70-8

81. O'Donovan A, Leech M, Gillham C. Assessment and management of radiotherapy induced toxicity in older patients. J Geriatr Oncol 2017;8:421-7.

82. Chin R, Fisher RJ, Smee RI, et al. Oropharyngeal cancer in the elderly. Int J Radiat Oncol Biol Phys 1995;32:1007-16.

83. Lusinchi A, Bourhis J, Wibault P, et al. Radiation therapy for head and neck cancers in the elderly. Int J Radiat Oncol Biol Phys 1990;18:819-23.

84. Strom TJ, Naghavi AO, Trotti AM, et al. Increased acute mortality with chemoradiotherapy for locally advanced head and neck cancer in patients $\geq 70$ years. J Geriatr Oncol 2017;8:50-5.

85. Nguyen NP, Godinez J, Shen W, et al. Is surgery indicated for elderly patients with early stage nonsmall cell lung cancer, in the era of stereotactic body radiotherapy? Medicine (Baltimore) 2016;95:e5212.

86. Takeda A, Sanuki N, Eriguchi T, et al. Stereotactic ablative body radiation therapy for octogenarians with non-small cell lung cancer. Int J Radiat Oncol Biol Phys 2013;86:257-63.

87. Wang P, Zhang D, Guo XG, et al. A propensity-matched analysis of surgery and stereotactic body radiotherapy for early stage non-small cell lung cancer in the elderly. Medicine (Baltimore) 2016;95:e5723.

88. Kreinbrink P, Blumenfeld P, Tolekidis G, et al. Lung stereotactic body radiation therapy (SBRT) for early-stage non-small cell lung cancer in the very elderly ( $\geq 80$ years old): Extremely safe and effective. J Geriatr Oncol 2017;8:351-5

89. Shinde A, Li R, Kim J, et al. Stereotactic body radiation therapy (SBRT) for early-stage lung cancer in the elderly. Semin Oncol 2018;45:210-9.

90. Lee K, Kim HO, Choi HK, et al. Real-world treatment patterns for patients 80 years and older with early lung cancer: a nationwide claims study. BMC Pulm Med 2018;18:127.

91. Shu Z, Dong B, Shi L, et al. Stereotactic body radiotherapy for elderly patients ( $\geq 75$ years) with earlystage non-small cell lung cancer. J Cancer Res Clin Oncol 2020;146:1263-71.

92. Haasbeek CJ, Lagerwaard FJ, Antonisse ME, et al. Stage I nonsmall cell lung cancer in patients aged $>$ or $=75$ years: outcomes after stereotactic radiotherapy. Cancer 2010;116:406-14.

93. Haehl E, Rühle A, David H, et al. Radiotherapy for geriatric head-and-neck cancer patients: what is the 
value of standard treatment in the elderly? Radiat Oncol 2020;15:31.

94. Sommers LW, Steenbakkers RJHM, Bijl HP, et al. Survival Patterns in Elderly Head and Neck Squamous Cell Carcinoma Patients Treated With Definitive Radiation Therapy. Int J Radiat Oncol Biol Phys 2017;98:793-801.

95. Bonomo P, Desideri I, Loi M, et al. Elderly patients affected by head and neck squamous cell carcinoma unfit for standard curative treatment: Is de-intensified, hypofractionated radiotherapy a feasible strategy? Oral Oncol 2017;74:142-7.

96. Belgioia L, Bacigalupo A, Missale F, et al. Individualized treatment of head neck squamous cell carcinoma patients aged 70 or older with radiotherapy alone or associated to cisplatin or cetuximab: impact of weekly radiation dose on loco-regional control. Med Oncol 2019;36:42.

97. Amini A, Jones BL, McDermott JD, et al. Survival outcomes with concurrent chemoradiation for elderly patients with locally advanced head and neck cancer according to the National Cancer Data Base. Cancer 2016;122:1533-43.

98. Belgioia L, Desideri I, Errico A, et al. Safety and efficacy of combined radiotherapy, immunotherapy and targeted agents in elderly patients: A literature review. Crit Rev Oncol Hematol 2019;133:163-70.

99. Mazzola R, Jereczek-Fossa BA, Franceschini D, et al. Oligometastasis and local ablation in the era of systemic

Cite this article as: Chau B, LaGuardia J, Hui C, Ye L, Xing Y, Massarelli E, Amini A. Narrative review of immunotherapy and radiation therapy in elderly patients. Transl Cancer Res 2021;10(5):2620-2631. doi: 10.21037/tcr-20-2637 targeted and immunotherapy. Radiat Oncol 2020;15:92.

100.Lazzari G, Terlizzi A, Porrazzo G, et al. Effective nivolumab sequential thoracic radiotherapy in elderly patients with advanced squamous cell lung cancer: did radiation therapy play a role? A case report. Onco Targets Ther 2018;11:4621-9.

101.Yan L, Wu M, Ba N, et al. Efficacy of dendritic cellcytokine-induced killer immunotherapy plus intensitymodulated radiation therapy in treating elderly patients with esophageal carcinoma. Genet Mol Res 2015;14:898-905.

102. Extermann M, Hurria A. Comprehensive geriatric assessment for older patients with cancer. J Clin Oncol 2007;25:1824-31.

103. Morris L, Thiruthaneeswaran N, Lehman M, et al. Are Future Radiation Oncologists Equipped With the Knowledge to Manage Elderly Patients With Cancer? Int J Radiat Oncol Biol Phys 2017;98:743-7.

104.Loh KP, Soto-Perez-de-Celis E, Hsu T, et al. What Every Oncologist Should Know About Geriatric Assessment for Older Patients With Cancer: Young International Society of Geriatric Oncology Position Paper. J Oncol Pract 2018;14:85-94.

105. Kirkhus L, Šaltytė Benth J, Rostoft S, et al. Geriatric assessment is superior to oncologists' clinical judgement in identifying frailty. Br J Cancer 2017;117:470-7. 